\title{
EQUICONTINUITY OF A GRAPH MAP
}

\section{TaIXIANg Sun, Yongping Zhang and XiaOyan Zhang}

Let $G$ be a graph, and $f: G \longrightarrow G$ be a continuous map with periodic points. In this paper we show that the following five statements are equivalent.

(1) $f$ is equicontinuous.

(2) There exists some positive integer $N$ such that $f^{N}$ is uniformly convergent.

(3) $f$ is $S$-equicontinuous for some positive integer sequence $S=\left\{n_{1}<n_{2}<\cdots\right\}$.

(4) $\Omega(x, f)=\omega(x, f)$ for every $x \in G$.

(5) $\sigma: \lim _{\lfloor}\{X, f\} \rightarrow \lim \{X, f\}$ is a periodic map.

\section{INTRODUCTION}

Let $\mathbf{N}$ (respectively $\mathbf{Z}^{+}$) denote the set of positive integers (respectively nonnegative integers ). Write $\mathbf{N}_{n}=\{1,2 \ldots, n\}$ and $\mathbf{Z}_{n}=\{0,1, \ldots, n\}$ for any $n \in \mathbf{N}$. For any compact metric space $(X, d)$, let $C^{0}(X)$ be the set of all continuous maps from $X$ to $X$. Suppose $f \in C^{0}(X), x \in X$ and $r>0$, write $B(x, r)=B(x, r, d)=\{y \in X: d(y, x)$ $<r\}, O(x, f)=\left\{f^{n}(x): n \in \mathbf{Z}^{+}\right\}, \omega(x, f)=\bigcap_{n=0}^{\infty} \overline{O\left(f^{n}(x), f\right)}$ and $\Omega(x, f)=\{y$ : there exist sequences $\left\{x_{i}\right\}$ in $X$ and $\left\{n_{i}\right\}$ in $\mathbf{N}$ such that $x_{i} \longrightarrow x, n_{i} \rightarrow \infty$ and $\left.f^{n_{i}}\left(x_{i}\right) \longrightarrow y\right\}$. $O(x, f)$ and $\omega(x, f)$ are called the orbit and the $\omega$-limit set of $x$ under $f$, respectively. For $n \in \mathrm{N}$, a point $x \in X$ is called a periodic point of $f$ with period $n$ (or an $n$-periodic point of $f)$ if $f^{n}(x)=x$ and $f^{k}(x) \neq x$ for each $k \in[0, n) \cap \mathbf{N}$. $x$ is called a fixed point of $f$ if $f(x)=x$. If $x \in \omega(x, f)$, then $x$ is called a recurrent point of $f$. Denote by $F(f), P_{n}(f)$ and $R(f)$ the set of all fixed points, $n$-periodic points and recurrent points of $f$, respectively. Write $P(f)=\bigcup_{n=1}^{\infty} P_{n}(f)$. We use int $A, \partial A, \bar{A}$ and $\# A$ to denote the interior, boundary, the closure and the cardinality of a subset $A$ of $X$, respectively. We also need the following definitions.

Definition 1: Let $S=\left\{n_{1}<n_{2}<\cdots\right\}$ be a subsequence of $\mathbf{N}$. $f \in C^{0}(X)$ is said to be $S$-equicontinuous if for any $\varepsilon>0$, there exists $\delta=\delta(\varepsilon)$ such that

Received 23rd August, 2004

This project was supported by NNSF of China (10361001, 10461001) and NSF of Guangxi (0447004).

Copyright Clearance Centre, Inc. Serial-fee code: 0004-9727/05 \$A2.00+0.00. 
$d\left(f^{n_{k}}(x), f^{n_{k}}(y)\right)<\varepsilon$ whenever $x, y \in X$ with $d(x, y)<\delta$ and $k \in \mathbf{N}$. If $S=\mathrm{N}$, then $f$ is said to be equicontinuous.

Definition 2: Let $f \in C^{0}(X)$ and $x \in X$. If there exists $y \in X$ such that $\lim _{n \rightarrow \infty} f^{n}(x)=y$, then $f$ is said to be convergent at $x$. If there exists $N \in \mathbf{N}$ such that $X=F\left(f^{N}\right)$, then $f$ is said to be a periodic map.

Let $\left\{X_{i}\right\}_{i=1}^{\infty}$ be a sequence of spaces, and $\left\{f_{i}\right\}_{i=1}^{\infty}$ a sequence of maps $f_{i}: X_{i+1} \longrightarrow X_{i}$, then the inverse limit of $\left\{X_{i}, f_{i}\right\}_{i=1}^{\infty}$, denoted by $\lim _{\lfloor}\left\{X_{i}, f_{i}\right\}$, is the subspace of the Cartesian product space $\prod_{i=1}^{\infty} X_{i}$ given by $\varliminf_{\lfloor}\left\{X_{i}, f_{i}\right\}=\left\{\left(x_{1}, x_{2}, \ldots\right): f_{i}\left(x_{i+1}\right)=x_{i}\right.$ for all $i \in \mathbf{N}\}$. When all the spaces $X_{i}$ are the same space $X$ and all the maps $f_{i}$ are same map $f$, we denote the inverse limit by $\varliminf_{\lfloor}\{X, f\}$ (see [10]).

Define $\sigma: \varliminf_{\lfloor}\{X, f\} \longrightarrow \lim \{X, f\}$ by

$$
\sigma:\left(\left(x_{0}, x_{1}, \ldots\right)\right)=\left(x_{1}, x_{2}, \ldots\right),
$$

which is called the one-sided shift map.

It is interesting to find some properties equivalent to equicontinuity ([1]). In [3], Blanchard, Host and Maass discussed topological complexity, and showed that a continuous surjection $f$ of a compact metric space $X$ is equicontinuous if and only if any finite open cover of $X$ under $f$ has bounded complexity.

On 1-dimentional spaces, one has some still finer results $[4,5,6,7,13]$. Sun in $[11,12]$ obtained necessary and sufficient conditions for equicontinuity of tree maps and $\sigma$-maps. In [8], Gu obtained necessary and sufficient conditions for equicontinuity of figure-eight map with a periodic point. Recently, Mai in [9] obtained the following theorem.

Theorem A. Let $G$ be a graph and $f \in C^{0}(G)$ with $P(f) \neq \emptyset$. Then $f$ is equicontinuous if and only if there exists $N \in \mathrm{N}$ such that $\bigcap_{n=1}^{\infty} f^{n}(G)=F\left(f^{N}\right)$.

By a graph we mean a compact connected one-dimensional polyhedron. In this paper we shall find some new equivalent conditions of equicontinuous graph maps. Our main result is the following theorem:

THEOREM 2. Let $G$ be a graph and $f \in C^{0}(G)$ with $P(f) \neq \emptyset$. Then the following five statements are equivalent.

(1) $f$ is equicontinuous.

(2) There exists $N \in \mathrm{N}$ such that $f^{N}$ is uniformly convergent.

(3) $f$ is $S$-equicontinuous for some subsequence $S=\left\{n_{1}<n_{2}<\cdots\right\}$ of $\mathrm{N}$.

(4) $\Omega(x, f)=\omega(x, f)$ for every $x \in G$.

(5) $\sigma: \lim \{X, f\} \longrightarrow \lim \{X, f\}$ is a periodic map. 


\section{EQUiCONTINUITY AND UNIFORM CONVERGENCE IN $C^{0}(X)$}

In this section we shall discuss the relation between equicontinuity and uniform convergence of continuous self-maps of a compact metric space.

THEOREM 1. Let $X$ is a compact metric space and $f \in C^{0}(X)$. Then the following three statements are equivalent:

(1) $f$ is uniformly convergent;

(2) $\bigcap_{n=1}^{\infty} f^{n}(X)=F(f)$ and $f$ is equicontinuous;

(3) $\bigcap_{n=1}^{\infty} f^{n}(X)=F(f)$ and $\Omega(x, f)=\omega(x, f)$ for every $x \in X$.

Proof: It is easy to see that $\Omega(x, f) \cup \omega(x, f) \subset \bigcap_{n=1}^{\infty} f^{n}(X)$ for every $x \in X$.

(2) $\Rightarrow(1)$ Suppose $\bigcap_{n=1}^{\infty} f^{n}(X)=F(f)$ and $f$ is equicontinuous. Let $x \in X$ and $a, b \in \omega(x, f)$, then $a, b \in F(f)$. Since $f$ is equicontinuous, for any $\varepsilon>0$, there exists $\delta>0$ such that $f^{n}(B(u, \delta)) \subset B\left(f^{n}(u), \varepsilon / 3\right)$ for every $u \in X$ and every $n \in \mathbf{N}$. Take $m \in \mathbf{N}$ such that $f^{m}(x) \in B(a, \delta)$, then $b \in \omega(x, f)=\omega\left(f^{m}(x), f\right) \subset B(a, \varepsilon)$. That is, $\{a\}=\omega(x, f)$, which implies that $f$ is convergent at $x$.

Choose $\left\{x_{1}, x_{2}, \ldots, x_{k}\right\} \subset X$ such that $\bigcup_{i=1}^{k} B\left(x_{i}, \delta\right)=X$. Then there exists $N \in \mathbf{N}$ such that

$$
d\left(f^{n}\left(x_{i}\right), f^{m}\left(x_{i}\right)\right)<\varepsilon / 3 \quad \text { for every } i \in \mathbf{N}_{k} \text { and any } n>m>N .
$$

For any $x \in X$, let $x \in B\left(x_{i}, \delta\right)$ for some $i \in \mathbf{N}_{k}$, then when $n>m>N$, we have

$$
d\left(f^{n}(x), f^{m}(x)\right)<d\left(f^{n}(x), f^{n}\left(x_{i}\right)\right)+d\left(f^{n}\left(x_{i}\right), f^{m}\left(x_{i}\right)\right)+d\left(f^{m}(x), f^{m}\left(x_{i}\right)\right)<\varepsilon .
$$

This implies $f$ is uniformly convergent.

$(2) \Rightarrow(3)$ : See $[1]$.

$(1) \Rightarrow(2)$ : Let $g(x)=\lim _{n \rightarrow \infty} f^{n}(x)$, then $g(x)$ is continuous. For any $\varepsilon>0$, there exist $N \in \mathbf{N}$ and $\delta>0$ such that

$$
d\left(f^{n}(x), g(x)\right)<\varepsilon / 3 \quad \text { for every } n>N \text { and every } x \in X,
$$

and

$$
g(B(x, \delta)) \subset B(g(x), \epsilon / 3) \quad \text { for every } x \in X
$$

and

$$
f^{i}(B(x, \delta)) \subset B\left(f^{i}(x), \varepsilon\right) \quad \text { for every } i \in \mathbf{N}_{N} \text { and every } x \in X .
$$

Thus we have

$$
f^{n}(B(x, \delta)) \subset B\left(f^{n}(x), \varepsilon\right) \quad \text { for any } n \in \mathbf{N} \text {. }
$$


This implies $f$ is equicontinuous.

Let $x \in \bigcap_{\substack{n=1 \\ \infty}}^{\infty} f^{n}(X)$, it follows from [9] that $x \in R(f)$. Then $x \in \omega(x, f)=\{x\}$, which implies $\bigcap_{n=1}^{\infty} f^{n}(X)=F(f)$.

$(3) \Rightarrow(2)$ It only needs to be shown that $f$ is equicontinuous. For given $x \in X$, let $x_{n} \rightarrow x, k_{n} \rightarrow \infty, f^{k_{n}}\left(x_{n}\right) \rightarrow a$ and $f^{k_{n}}(x) \rightarrow b$, then $a \in \Omega(x, f)=\omega(x, f)$ and $a, b \in F(f)$. Hence there exists $t_{n} \rightarrow \infty$ such that $t_{n}-k_{n}>n$ and $f^{t_{n}-k_{n}}\left(f^{k_{n}}(x)\right)$ $=f^{t_{n}}(x) \rightarrow a$, which implies $a \in \omega(b, f)=\{b\}$. That is, $f$ is equicontinuous.

\section{Proof of TheOREM 2}

Let $G$ be a graph. For every $x \in G$, there exist a positive number $\varepsilon>0$ and some $n$-star $X_{n}=\left\{z: z^{n} \in[0,1], z\right.$ is a complex number $\}$ ([2]) such that for every $0<\delta \leqslant \varepsilon$, there exists a homeomorphism $f: \overline{B(x, \delta)} \longrightarrow X_{n}$, such $B(x, \delta)$ are said to be a $n$-starneighbourhoods of $x$. Write $V(x)=n$. If $V(x) \geqslant 3$, we call $x$ a branched point of $G$. Let $T$ ([2]) be a subtree of $G$ and $a, b \in T$, we use $[a, b]_{T}$ (or $[b, a]_{T}$ ) to denote the smallest connected subset of $T$ containing $a, b$. Write $[a, b)_{T}=[a, b]_{T}-\{b\},(a, b)_{T}=[a, b)_{T}-\{a\}$.

In what follows we let $B(G)=\left\{x_{1}, x_{2}, \ldots, x_{l}\right\}$ be the set of all branched points of $G$, and $G-B(G)$ have $p$ connected components. Put $u=V\left(x_{1}\right)+V\left(x_{2}\right)+\cdot+V\left(x_{l}\right)+4 p$ and $M=u$ !. Let $S=\left\{n_{1}<n_{2}<\cdots\right\}$ be a subsequence of $\mathbf{N}$.

LEMMA 1. Let $f \in C^{0}(G)$ and $m \in \mathbf{N}$, then

(1) $f$ is equicontinuous if and only if $f^{m}$ is equicontinuous, and

(2) if $f$ is $S$-equicontinuous, then $g=f^{m}$ is $S_{1}$ - equicontinuous for some subsequence $S_{1}$ of $\mathrm{N}$.

ProOF: (1) See [9].

(2) Let $f$ be $S$-equicontinuous. Then by choosing a subsequence we can assume that there exists $r \in \mathbf{Z}_{m-1}$ such that $n_{i}=s_{i} m+r$ for any $i \in \mathbf{N}$. Since $G$ is compact, for any $\varepsilon>0$, there exists $\delta_{1}>0$ such that

$$
d\left(f^{i}(u), f^{i}(v)\right)<\varepsilon \quad \text { whenever } d(u, v)<\delta_{1} \text { and } i \in \mathbf{Z}_{m}
$$

Since $f$ is $S$-equicontinuous, there exists $\delta$ such that

$$
d\left(f^{s_{i} m+r}(u), f^{s_{i} m+r}(v)\right)<\delta_{1} \quad \text { whenever } d(u, v)<\delta \text { and } i \in \mathbf{N} .
$$

Hence

$$
d\left(g^{s_{i}+1}(u), g^{s_{i}+1}(v)\right)<\varepsilon \quad \text { whenever } d(u, v)<\delta \text { and } i \in \mathbf{N} .
$$

This implies that $g$ is $S_{1}=\left\{s_{1}+1, s_{2}+1, \ldots\right\}$-equicontinuous.

LEMMA 2. Let $f \in C^{0}(G)$, and $X=\bigcap_{n=1}^{\infty} f^{n}(G)$. If one of following two conditions holds, 
(1) $f$ is $S$-equicontinuous, or

(2) $\Omega(x, f)=\omega(x, f)$ for every $x \in X$;

then for any given $m \in \mathbf{N}, X \subset \omega\left(\left.f^{m}\right|_{X}\right)$.

Proof: Let $g=f^{m}$. Since $X$ is compact, we have $g(X)=X$ and $X$ is a connected closed subset of $G$. For given $y_{0} \in X$, there exist points $y_{1}, y_{2}, \ldots$ in $X$ such that $g\left(y_{n}\right)=y_{n-1}$ for every $n \in \mathbf{N}$.

(1) If $f$ is $S$-equicontinuous, then by Lemma 1 there exists a subsequence $S_{1}=\left\{s_{1}<s_{2}<\cdots\right\}$ of $\mathbf{N}$ such that $g$ is $S_{1}$ - equicontinuous. Therefore for any $\varepsilon>0$, there exists $\delta>0$ such that $d\left(g^{s_{k}}(u), g^{s_{k}}(v)\right)<\varepsilon$ whenever $d(u, v)<\delta$ and $k \in \mathbf{N}$. Since $X$ is compact, there exists a subsequence $0<k_{1}<k_{2}<\cdots$ of $\mathbf{N}$ and $y \in X$ such that $y_{s_{k_{j}}} \longrightarrow y$. Then $d\left(g^{s_{k_{j}}}\left(y_{s_{k_{j}}}\right), g^{s_{k_{j}}}(y)\right)=d\left(y_{0}, g^{s_{k_{j}}}(y)\right)<\varepsilon$ for some $s_{k_{j}} \in \mathbf{N}$. Thus $y_{0} \in \omega(y, g)$.

(2) If $\Omega(x, f)=\omega(x, f)$ for every $x \in X$, then by choosing subsequence we can assume that there exists a subsequence $0<k_{1}<k_{2}<\cdots$ of $\mathbf{N}$ and $y \in X$ such that $y_{k_{j}} \longrightarrow y$ since $X$ is compact. Thus $y_{0} \in \Omega(y, f)=\omega(y, f)=\bigcup_{i=0}^{m-1} \omega\left(f^{i}(y), g\right)$.

Lemma 3. Let $f \in C^{0}(G)$ with $P(f) \neq \emptyset$ and $X=\bigcap_{n=1}^{\infty} f^{n}(G)$. If one of following two conditions holds,

(1) $f$ is $S$-equicontinuous; or

(2) $\Omega(x, f)=\omega(x, f)$ for every $x \in X$;

then $X=F\left(f^{\tau M}\right)$. Where $\tau$ is the smallest period of the periodic points of $f$.

Proof: Let $g=f^{\tau}$. Obviously $F\left(g^{M}\right) \subset X$. Now we show $X \subset F\left(g^{M}\right)$.

Assume on the contrary that $X-F\left(g^{M}\right) \neq \emptyset$. Take $p \in F(g)$ and let $K$ be the connected component of $F\left(g^{M}\right)$ containing $p$, then $K$ is a closed subset of $X, g(K)=K$ and $\partial K \cap \partial(X-K) \neq \emptyset$.

Claim 1. $g(\partial K \cap \partial(X-K)) \subset \partial K \cap \partial(X-K)$.

Proof of Claim 1: Assume on the contrary that there exists $a \in \partial K \cap \partial(X-K)$ such that $g(a) \notin \partial K \cap \partial(X-K)$. Then we can choose a neighbourhood $U$ of $a$ such that $U \cap(X-K) \neq \emptyset$ and $g(U \cap(X-K)) \subset K$. Thus $U \cap(X-K) \not \subset \omega\left(\left.g\right|_{X}\right)$ since $g(K)=K$, which contradicts Lemma 2. Claim 1 is proven.

Take $a_{0} \in \partial K \cap \partial(X-K)$. Let $s$ be the period of $a_{0}$ under $g$ and $V_{i}$ be a $k_{i}$-starneighbourhood of $g^{i}\left(a_{0}\right)\left(i \in \mathbf{Z}_{s-1}\right)$. We can assume $k_{0}=\min \left\{k_{i}: i \in \mathbf{Z}_{s-1}\right\}$. Choose $0<\delta_{1}<\delta_{2}<\cdots<\delta_{k_{0}+2}$ such that

$$
g^{s}\left(B\left(a_{0}, \delta_{i}\right)\right) \subset B\left(a_{0}, \delta_{i+1}\right) \subset V_{0} \quad\left(i \in \mathrm{N}_{k_{0}+1}\right) .
$$

CLAIM 2. If there exist $y \in B\left(a_{0}, \delta_{k_{0}+1}\right)$ and $k \in \mathbf{N}$ such that $\left\{g^{i s}(y): i \in \mathbf{Z}_{k}\right\}$ $\subset B\left(a_{0}, \delta_{k_{0}+1}\right)$ and $y, g^{k s}(y)$ is contained in same connected component $L$ of $B\left(a_{0}, \delta_{k_{0}+1}\right)$ $-\left\{a_{0}\right\}$, then $g^{k s}(y) \in\left(a_{0}, y\right]_{L}$. 
ProOF OF Claim 2: Suppose that $y_{0}=y \in\left(a_{0}, g^{k s}(y)\right)_{L}$. Then there exist points $y_{1}, y_{2}, \ldots$ in $L$ such that $y_{n} \in\left(a_{0}, y_{n-1}\right)_{L}$ and $g^{k s}\left(y_{n}\right)=y_{n-1}(n \in \mathrm{N})$. Let $y_{n} \rightarrow v \in F\left(g^{k s}\right)$, then $y_{0} \in \Omega(v, f)-\omega(v, f)$ and $d\left(g^{k s n}\left(y_{n}\right), g^{k s n}(v)\right)=d\left(y_{0}, v\right)>0$ for any $n \in \mathrm{N}$, which implies that $g^{k s}$ is not $S$-equicontinuous for any subsequence $S$ of $\mathrm{N}$. A contradiction. Claim 2 is proven.

Claim 3. Let $y \in B\left(a_{0}, \delta_{1}\right)$, then $O\left(y, g^{s}\right) \subset B\left(a_{0}, \delta_{k_{0}+1}\right)$.

ProOF of ClaIM 3: Assume on the contrary that $O\left(y, g^{s}\right) \not \subset B\left(a_{0}, \delta_{k_{0}+1}\right)$. Let $B_{1}$ be the connected component of $B\left(a_{0}, \delta_{k_{0}+1}\right)-\left\{a_{0}\right\}$ containing $y$ and $r_{1}=\min \{i$ : $\left.g^{i s}(y) \notin B_{1}\right\}$. It follows from Claim 2 that $\left\{y, \ldots, g^{\left(r_{1}-1\right) s}(y)\right\} \subset B\left(a_{0}, \delta_{1}\right)$. Let $B_{2}$ be the connected component of $B\left(a_{0}, \delta_{k_{0}+1}\right)-\left\{a_{0}\right\}$ containing $g^{r_{1} s}(y)$ and $r_{2}=\min \left\{i: g^{i s}(y)\right.$ $\left.\notin B_{2} \cup B_{1}\right\}$. Again it follows from Claim 2 that $\left\{y, \ldots, g^{\left(r_{2}-1\right) s}(y)\right\} \subset B\left(a_{0}, \delta_{2}\right)$. Continuing on, we inductively define $0=r_{0}<r_{1} \leqslant r_{2} \leqslant \cdots \leqslant r_{k_{0}}$ and the connected components $B_{1}, B_{2}, \ldots, B_{k_{0}}$ of $B\left(a_{0}, \delta_{k_{0}+1}\right)-\left\{a_{0}\right\}$ such that

(i) $r_{j}=\min \left\{i: g^{i s}(y) \notin \bigcup_{\lambda=1}^{j} B_{\lambda}\right\}$ for every $j \in \mathbf{N}_{k_{0}}$;

(ii) $B_{j}$ be the connected component of $B\left(a_{0}, \delta_{k_{0}+1}\right)-\left\{a_{0}\right\}$ containing $g^{r_{j-1} s}(y)$ for every $j \in \mathbf{N}_{k_{0}}$;

(iii) $\left\{y, \ldots, g^{\left(r_{j}-1\right) s}(y)\right\} \subset B\left(a_{0}, \delta_{j}\right)$ for every $j \in \mathbf{N}_{k_{0}}$.

Hence $g^{r_{k_{0}} s}(y) \in B\left(a_{0}, \delta_{k_{0}+1}\right)$ since $g^{s}\left(B\left(a_{0}, \delta_{k_{0}}\right)\right) \subset B\left(a_{0}, \delta_{k_{0}+1}\right)$, which contradicts the definition of $r_{k_{0}}$. Hence $O\left(x, g^{s}\right) \subset B\left(a_{0}, \delta_{k_{0}+1}\right)$. Claim 3 is proven.

Claim 4. $\quad \omega\left(B\left(a_{0}, \delta_{1}\right) \cap(X-K), g\right) \subset F\left(g^{M}\right)$.

Proof of Claim 4: Let $y \in B\left(a_{0}, \delta_{1}\right) \cap(X-K)$, it follows from Claim 3 that $O\left(y, g^{s}\right) \subset B\left(a_{0}, \delta_{k_{0}+1}\right)$.

If $g^{i s}(y) \in K$ for some $i \in \mathbf{N}$, then $\omega\left(y, g^{s}\right) \subset F\left(g^{M}\right)$ and $\omega\left(g^{i}(y), g^{s}\right)$ $=g^{i}\left(\omega\left(y, g^{s}\right)\right) \subset g^{i}\left(F\left(g^{M}\right)\right) \subset F\left(g^{M}\right)$, which implies $\omega(y, g) \subset F\left(g^{M}\right)$.

If $O\left(y, g^{s}\right) \cap K=\emptyset$, then it follows from Claim 2 that $\#\left(\omega\left(y, g^{s}\right)\right)=r \leqslant k_{0}$ and $\omega\left(y, g^{s}\right) \subset F\left(g^{s r}\right)$. Thus $\omega\left(y, g^{s}\right) \subset F\left(g^{M}\right)$ and $\omega(y, g) \subset F\left(g^{M}\right)$. Claim 4 is proven. $\square$

Let $y \in B\left(a_{0}, \delta_{1}\right) \cap(X-K)$, it follows from Lemma 2 that there exists $x \in X$ such that $y \in \omega(x, g)$. Choose $m \in \mathbf{N}$ such that $g^{m}(x) \in B\left(a_{0}, \delta_{1}\right) \cap(X-K)$, then $y \in \omega(x, g)=\omega\left(g^{m}(x), g\right)$. By Claim 4 we have $y \in F\left(g^{M}\right)$. Hence $B\left(a_{0}, \delta_{1}\right) \cap(X-K)$ $\subset F\left(g^{M}\right)$, which implies $B\left(a_{0}, \delta_{0}\right) \cap(X-K) \subset K$, a contradiction. Lemma 3 is proven. $\square$

Proof of Theorem 2. (1) $\Leftrightarrow$ (2) is from Theorem A, Theorem 1 and Lemma 1.

$(1) \Leftrightarrow(3,4)$ is from $[1$, Theorem 2.3], Theorem $A$ and Lemma 3 .

$(1) \Rightarrow(5)$ Suppose $f$ is equicontinuous. It follows from Theorem A that $\bigcap_{n=1}^{\infty} f^{n}(G)$ $=F\left(f^{N}\right)$ for some $N \in \mathbf{N}$. Let $x=\left(x_{0}, x_{1}, \ldots\right) \in \lim _{\underset{\infty}{ }}\{X, f\}$, then for given $i \in \mathbf{Z}^{+}$, we have $x_{i}=f^{n}\left(x_{i+n}\right)$ for all $n \in \mathbf{N}$. Thus $x_{i} \in \bigcap_{n=1}^{\infty} f^{n}(G)=F\left(f^{N}\right)$, which implies $\sigma^{N}(x)=x$ for all $x=\left(x_{0}, x_{1}, \ldots\right) \in \lim \{X, f\}$. 
(5) $\Rightarrow(1)$ Suppose there exists $K \in \mathrm{N}$ such that $\sigma^{K}(x)=x$ for all $x \in \lim \{X, f\}$. Let $y \in X=\bigcap_{n=1}^{\infty} f^{n}(G)$. Since $f(X)=X$, there exist points $y_{1}=y, y_{2}, \ldots$ in $X$ such that $f\left(y_{i+1}\right)=y_{i}$ for all $i \in \mathbf{N}$, thus $x=\left(y_{1}, y_{2}, \ldots\right) \in \varliminf_{\lfloor}\{X, f\}, \sigma^{K}(x)=x$, which implies $y \in F\left(f^{K}\right)$. By Theorem A we know that $f$ is equicontinuous since $\bigcap_{n=1}^{\infty} f^{n}(G) \subset F\left(f^{K}\right)$.

\section{REFERENCES}

[1] E. Akin, J. Auslander and K. Berg, 'When is transitive map chaotic?', in Convergence in Ergodic Theory and Probability (Walter de Gruyter and Co., Berlin, New York, 1996), pp. 25-40.

[2] L. Alseda and J.M. Moreno, 'Linear orderings and the full periodicity kernel for the n-star', J. Math. Anal. Appl. 180 (1993), 599-616.

[3] F. Blanchard, B. Host and A. Maass, 'Topological complexity', Ergodic Theory Dynamical Systems 20 (2000), 641-662.

[4] A.M. Blokh, 'The set of all iterates is nowhere dense in $C([0,1],[0,1])$ ', Trans. Amer. Math. Soc. 333 (992), 787-798.

[5] W. Boyce, ' $\Gamma$-compact maps on an interval and fixed points', Trans. Amer. Math. Soc. 160 (1971), 87-102.

[6] A.M. Bruckner and T. Hu, 'Equicontinuity of iterates of an interval map', Tamkang J. Math. 21 (1990), 287-294.

[7] J. Cano, 'Common fixed points for a class of commuting mappings on an interval', Trans. Amer. Math. Soc. 86 (1982), 336-338.

(8] R. Gu, 'Equicontinuity of maps on figure-eight space', Southeast Asian Bull. Math. 25 (2001), 413-419.

[9] J. Mai, 'The structure of equicontinuous maps', Trans. Amer. Math. Soc. 355 (2003), 4125-4136.

[10] S.B. Nadler, Continuum theory, Pure and Appl. Math. 158 (Marcle Dekker, Inc., New York, 1992).

[11] T. Sun, 'Equicontinuity of $\sigma$-maps', Pure Appl. Math. (Xi'an) 16 (2000), 9-14.

[12] T. Sun, 'Equicontinuity of tree maps', J. Math. Study 34 (2001), 125-130.

[13] A. Valaristos, 'Equicontinuity of iterates of circle maps', Internat. J. Math. Math. Sci. 21 (1998), 453-458.

Department of Mathematics

Guangxi University

Nanning

Guangxi 530004

People's Republic of China

e-mail: stxhql@gxu.edu.cn 